\title{
Gallbladder Adenosquamous Cancer with Situs Inversus Totalis: A Case Report and Literature Review
}

\author{
Junming Huang ${ }^{1, *}$ \\ Hanjin Yang ${ }^{2, *}$ \\ Meng Wang' \\ Xinyu Zhao' \\ Shiyi Shao' \\ Fu Zhang' \\ Risheng Que' \\ Qida $\mathrm{Hu} \mathbb{1 D}^{\prime}$ \\ Tingbo Liang' \\ 'Department of Hepatobiliary and \\ Pancreatic Surgery, First Affiliated \\ Hospital, Zhejiang University School of \\ Medicine, Hangzhou, 310006, People's \\ Republic of China; ${ }^{2}$ Department of \\ Pathology, First Affiliated Hospital, \\ Zhejiang University School of Medicine, \\ Hangzhou, 310006, People's Republic of \\ China
}

*These authors contributed equally to this work
Correspondence: Qida Hu

Department of Hepatobiliary and Pancreatic Surgery, First Affiliated Hospital, Zhejiang University School of Medicine, 79 Qingchun Road, Hangzhou, 310006, People's Republic of China Email huqida@zju.edu.cn

Tingbo Liang

Department of Hepatobiliary and Pancreatic Surgery, First Affiliated Hospital, Zhejiang University School of Medicine, 79 Qingchun Road, Hangzhou, 310006, People's Republic of China Email liangtingbo@zju.edu.cn
Background: Situs inversus totalis (SIT) is a rare genetic congenital disease, characterized with complete right-to-left inversion of all the internal organs. We herein describe a meaningful case which was diagnosed as gallbladder adenosquamous carcinoma, a rare histology type of gallbladder cancer, with SIT.

Case Presentation: A 59-year-old Chinese woman was admitted for persistent epigastric distention and intermittent abdominal pain. The abdominal CT scan revealed a huge mass at the gallbladder bottom, involving the adjacent transverse colon and liver. En-bloc radical resection of the gallbladder cancer, including partial colectomy and hepatectomy with regional node dissection, followed by colocolostomy and Roux-en-Y choledochojejunostomy, was successfully performed. Pathology analysis indicated an adenosquamous carcinoma with positive adenocarcinoma markers (CK7, CK19) and squamous carcinoma markers (CK5/6, P63).

Conclusion: The SIT anomaly might increase the risk of malignancies by sharing genome mutations, suggesting the importance of surveillance in the SIT settings.

Keywords: situs inversus totalis, gallbladder adenosquamous carcinoma

\section{Background}

Situs inversus totalis (SIT) is a rare genetic predisposition where the organs in the chest and abdomen are positioned in a mirror manner from their normal location to the other side of the body. The incidence of SIT is approximately $1 / 8000$ to $1 /$ 25,000 in live births. ${ }^{1,2}$ Although the exact etiology of SIT is unclear, several mutations, such as DNAH11 and Nme7, are closely related to SIT. ${ }^{3,4}$ SIT does not seriously affect most organ functions and usually shows no significant symptoms, except that patients with ciliary dyskinesia might present with obvious mobility dysfunction. ${ }^{5}$ Therefore, SIT is found occasionally in routine imaging studies in most circumstances.

Due to the altered anatomical position of the vessels and organs in SIT, anatomical dissection and surgical resection proposed greater technical challenges to the surgeons. ${ }^{6}$ So far, there have been several excellent cases of laparoscopic cholecystectomy in patients with SIT through advanced surgical techniques. ${ }^{7}$ On the contrary, only a few cases of successful surgical treatments in SIT patients of esophageal cancer, pancreatic cancer, or ovarian cancer have been reported. ${ }^{8-10}$ To our knowledge, report of SIT combined with gallbladder cancer has been rare. Here, 
we describe a successful case of radical resection of gallbladder cancer in a SIT patient.

\section{Case Presentation}

A 59-year-old Chinese woman was admitted to our hospital due to persistent epigastric distention and intermittent abdominal pain for 10 days. The patient had a 10-year history of gallbladder stones. Physical examination revealed right upper abdomen tenderness. All the tumor markers were in normal ranges. Initial chest X-ray scan found a mirror-image dextrocardia (Figure 1A), and further computed tomography (CT) revealed a complete, right-to-left reverse transposition of the organs in the thoracic cavity and the abdomen (Figure 1B and C), confirming her congenital anomaly of SIT.

A contrasted CT scan revealed a $6.3 \mathrm{~cm} \times 4.5 \mathrm{~cm}$ mass at the gallbladder bottom, with involvement of the transverse colon and the left lateral liver (Figure 2A and B). No other distant metastasis or lymph node enlargement was observed. The initial diagnosis was gallbladder cancer at stage IIIA (cT3N0M0) according to the AJCC staging guideline. ${ }^{11}$ Multiple vascular variations were also seen in addition to the right-to-left reversal anomaly (Figure 2C). Specifically, the celiac trunk divided into the splenic artery and the left gastric artery, while the common hepatic artery originates from the superior mesenteric artery. Polysplenia syndrome is defined as the existence of multiple, two to six, spleens similar in size, which are different from the accessory spleen. ${ }^{12}$ Until now, it is not clear whether a relationship exists between polysplenia syndrome and SIT. The coexistence of polysplenia syndrome is common in SIT, for instance in our case.

The multidisciplinary board considered the clinical diagnosis of resectable gallbladder cancer with SIT, and suggested surgical resection. En-bloc radical resection of the gallbladder cancer, including partial colectomy and hepatectomy with regional node dissection, followed by colocolostomy and Roux-en-Y choledochojejunostomy, was successfully performed. The whole operation took around 5 hours, and the estimated blood loss was $100 \mathrm{~mL}$. Gross anatomy showed multiple gallstones in the gallbladder and a huge tumor at the gallbladder bottom, invading the transverse colon and the liver (Figure 3A and B). Final pathology revealed a moderately differentiated adenosquamous carcinoma of the gallbladder, invading the submucosa of the transverse colon (Figure 3C and D), further confirmed by immunohistochemical staining showing positive P63, P53, CK5/6, CK19, CK7, CK20, and CDX2, and negative ERBR expression (Figure 4). All the 10 dissected lymph nodes were negative. The pathological stage of this patient is pT3N0M0. The patient recovered well after surgery. Two months post the surgical procedure, she started adjuvant gemcitabine plus cisplatin regimen. She received 5 cycles of chemotherapy and was free of recurrence at 5 months post operation.

\section{Discussion and Conclusions}

The exact etiology of SIT remains unclear, majorly caused by genetic mutations and chromosomal abnormalities. ${ }^{3,13}$ As SIT itself does not seriously affect the function of organs, most patients will not have any abnormal feeling and it is mainly found during radiological examinations. However, the incidence of cardiovascular, hepatobiliary, and spleen malformations, accompanied with anomalies of abdominal vessels origination or distribution, markedly increases in SIT patients. ${ }^{14,15}$ In our case, two major anatomical malformations were presented. One malformation was the vascular variation. In normal circumstances, the celiac trunk divides into the common hepatic artery, the splenic artery, and the left gastric artery. In this patient,

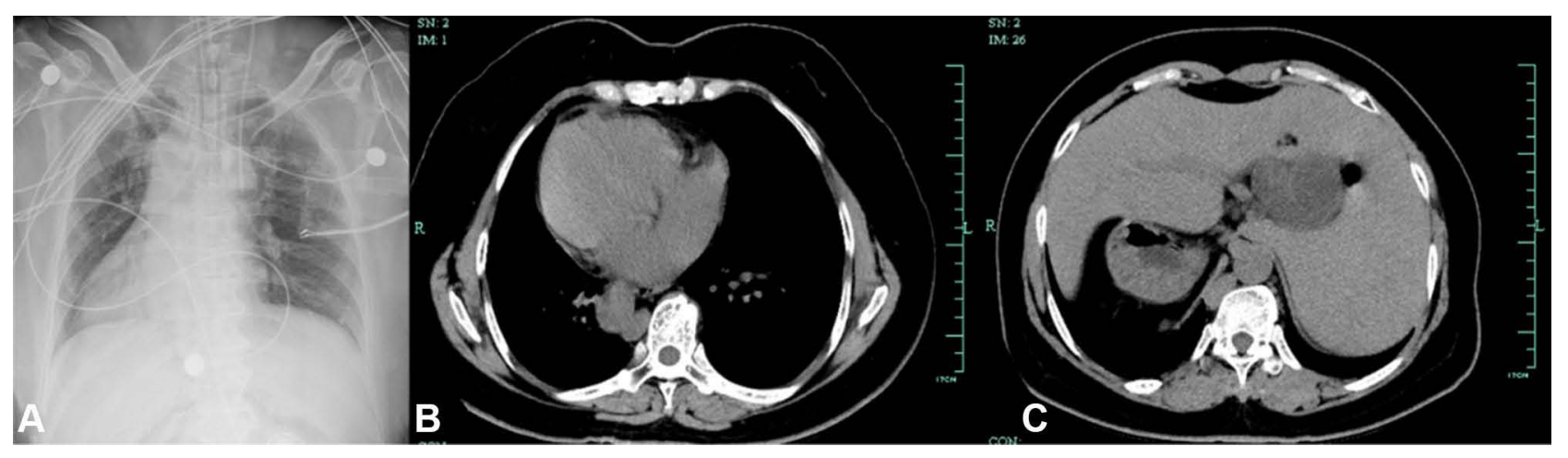

Figure I Radiology studies revealed SIT anomaly. (A) Dextrocardia in the chest X-ray image, right-to-left reverse transposition in (B) thoracic and (C) abdominal computed tomography (CT) images. 

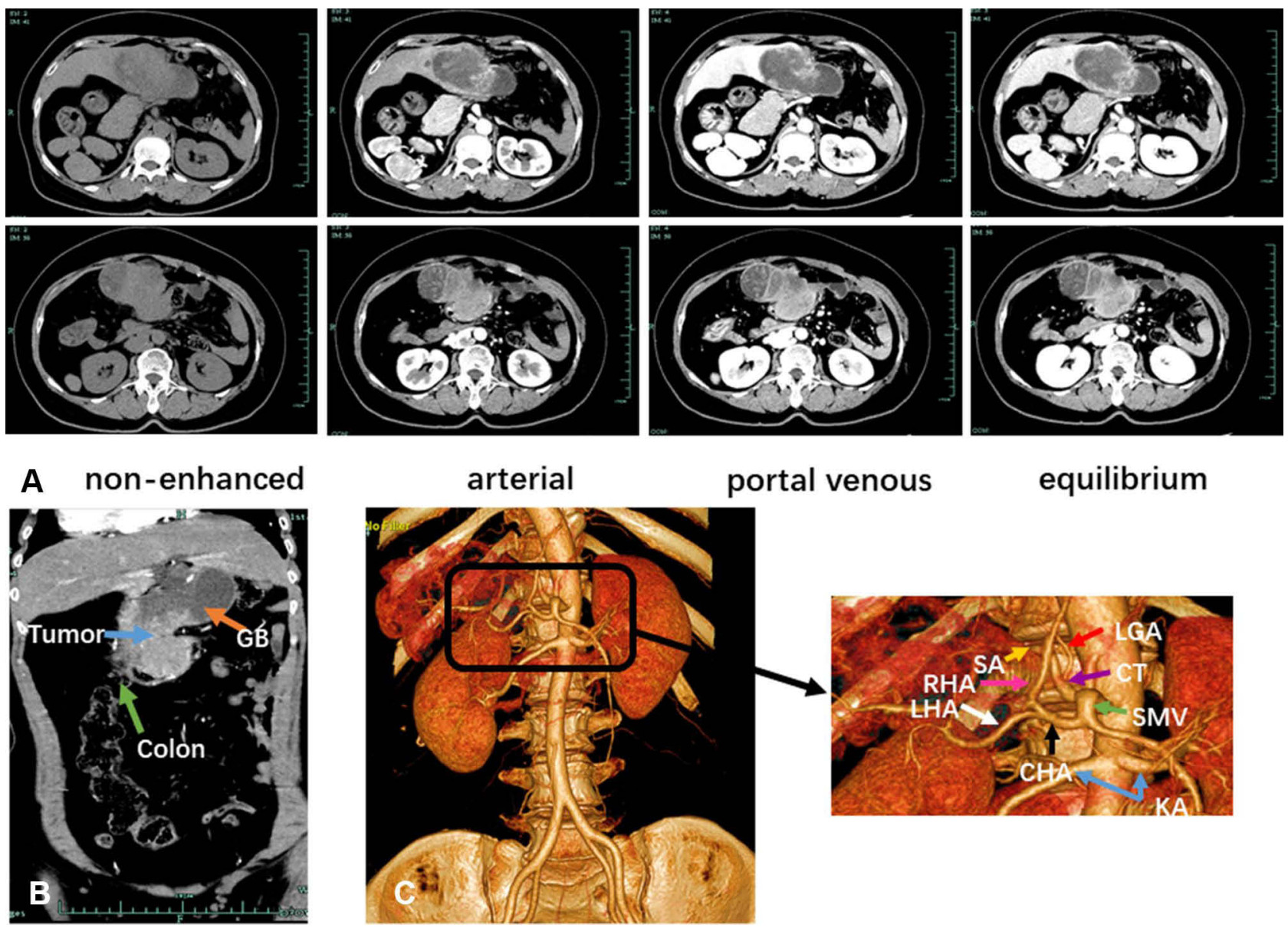

\section{equilibrium}

Figure 2 Preoperative CT scan indicated the diagnosis of gallbladder cancer in the SIT setting. (A) The CT images in the non-enhanced, arterial, portal venous, and equilibrium phases. (B) The coronal view showing tumor's involvement of transverse colon. (C) The CT angiography demonstrating significant vascular abnormalities. Abbreviations: GB, gallbladder; SMA, superior mesenteric artery; CT, celiac trunk; CHA, common hepatic artery; LGA, left gastric artery; SA, splenic artery; RHA, right hepatic artery; LHA, left hepatic artery; RA, renal artery.

the celiac trunk only divides to the splenic artery and the left gastric artery, while the common hepatic artery originates from the superior mesenteric artery. The other malformation was polysplenia syndrome, where three spleens with a similar size were presented. Polysplenia syndrome is often associated with multiple visceral and vascular abnormalities. ${ }^{16}$ These two malformations brought higher risks of intraoperative complications associated with incomprehension of the anatomical variations. In this case, a right-handed surgeon was also in the right side on the patient, although the patient was in the SIT setting. The source and destination of all blood vessels, especially arteries must be cleared before ligation and disconnection, since huge changes of the structure and location of vascellum.

Notably, malignancy is also a potential outcome of SIT since the two diseases might share certain mutations in several signaling pathways. The DNAH11 (axonemal heavy chain dynein type 11) gene mutations were found to cause SIT in mice, which was further confirmed in SIT patients. ${ }^{15}$ Meanwhile, the DNAH11 mutations are also associated with esophageal squamous cell carcinoma, ovarian cancer, and breast cancer. ${ }^{17,18}$ Another DNAH family member DNAH5, a SIT-related gene, was found to be mutated in several malignancies. ${ }^{19-21}$ Inversin, whose mutations cause an autosomal recessive cystic disorder characterized by SIT, functions as a molecular switch between Wnt signaling pathways, ${ }^{22}$ as the Wnt pathways has close relationship with carcinogenesis including development of gallbladder cancer. ${ }^{23,24}$

Therefore, it is possible that the mutations in SIT also results in malignancy. However, no specific shared mutation has been verified between SIT and malignancy yet.

Previous studies have demonstrated that SIT patients might develop malignancies like esophageal cancer, pancreatic cancer, and ovarian cancer. In our case, we showed 

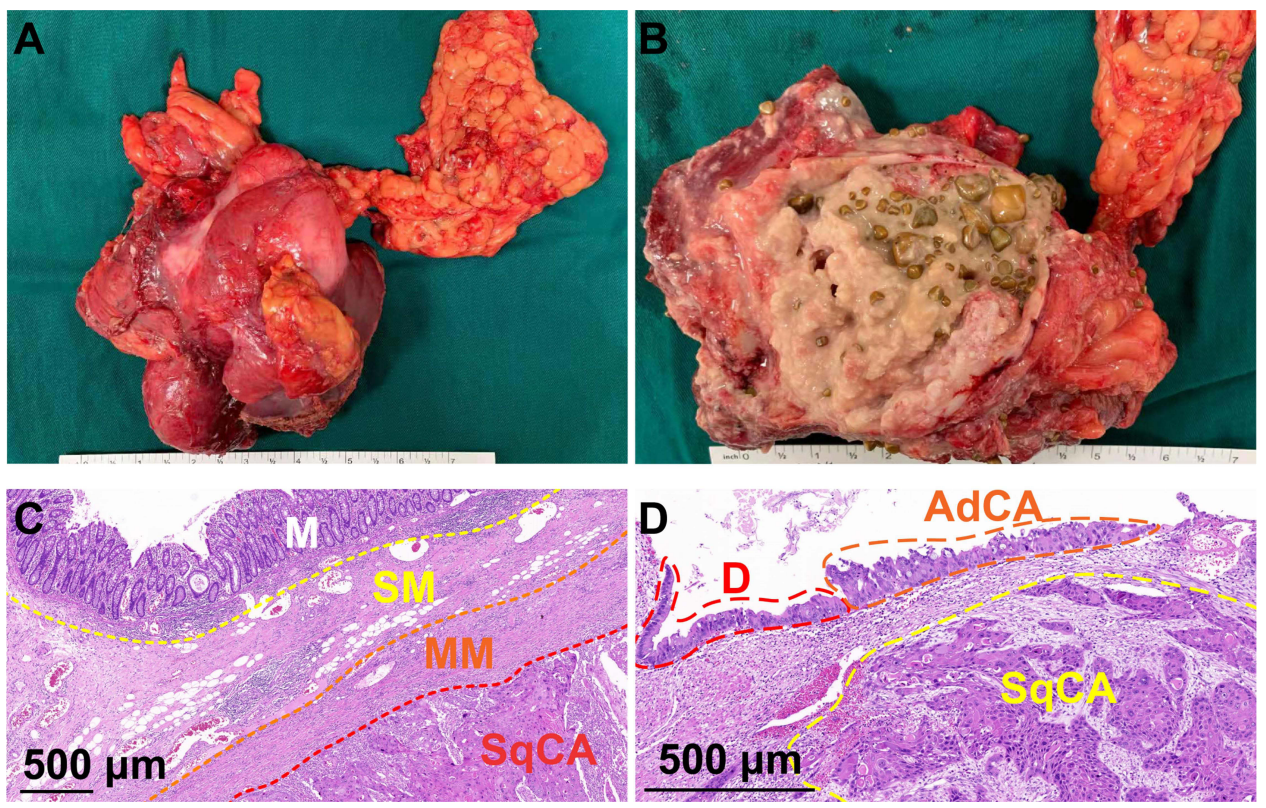

Figure 3 Pathology studies confirmed the diagnosis of gallbladder cancer. (A) The resected sample with adjacent colon and liver tissues. (B) Cross-sectional profile showing huge gallbladder mass and multiple gallstones. (C) $\mathrm{H} \& \mathrm{E}$ staining showing tumor's involvement of colon. (D) $\mathrm{H}$ \& $\mathrm{E}$ staining of gallbladder adenosquamous carcinoma.

Abbreviations: M, mucosa layer; SM, submucosa layer; MM, muscular layer; SqCA, squamous carcinoma; D, dysplasia in gallbladder glandular epithelium; AdCA, adenocarcinoma.

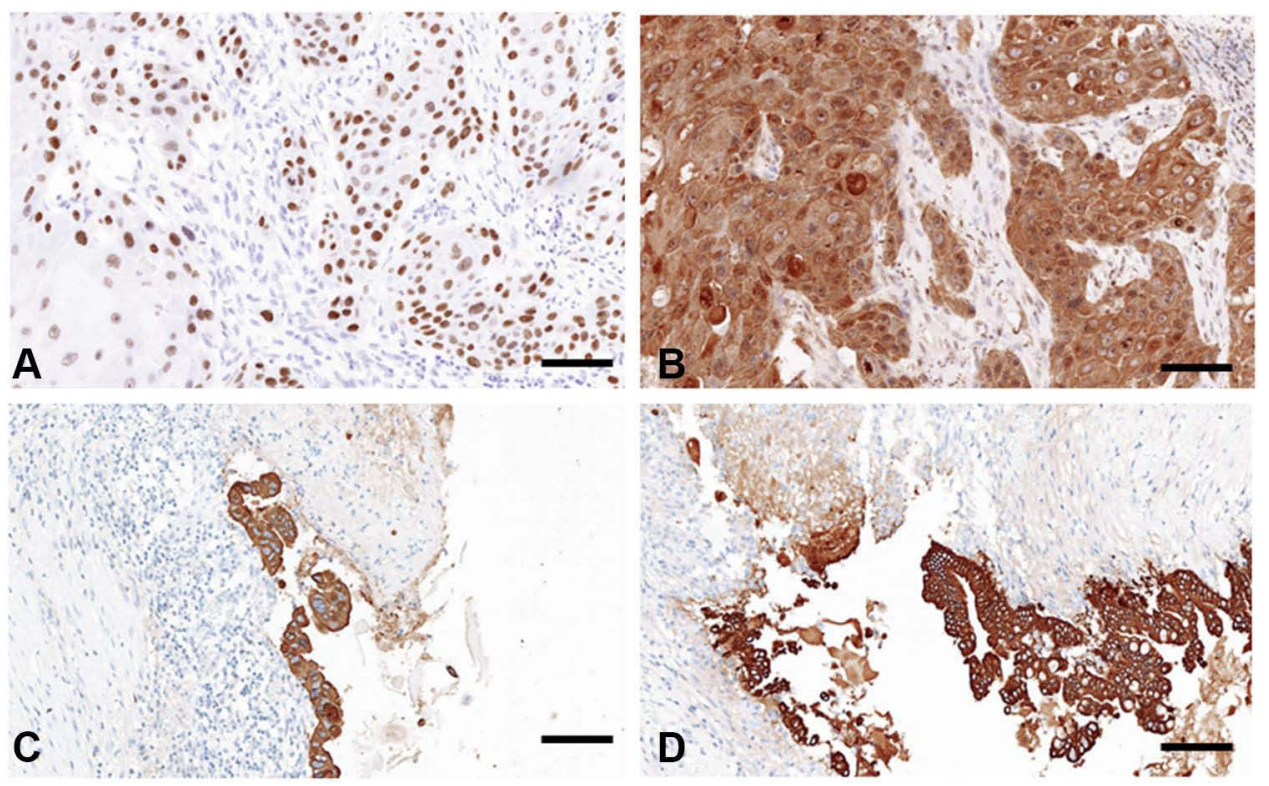

Figure 4 Immunohistochemistry assays suggested (A) positive P63, (B) positive CK5/6, (C) positive CK7, and (D) positive CKI9 expression. The scale bars indicate I00 $\mu$ m.

that gallbladder carcinoma, a highly malignant disease with poor prognosis, ${ }^{25}$ could also be an unfortunate outcome of SIT. Specifically, our pathology diagnosis is adenosquamous carcinoma, a relatively rare type across gallbladder malignancies, ${ }^{26,27}$ especially in a SIT setting.
Compared to adenocarcinoma, adenosquamous carcinoma is likely to develop a more advanced stage with rapid progression and declined prognosis. ${ }^{28}$ Optimistically, the survival data of resectable adenosquamous gallbladder cancer is comparable to that of gallbladder 
adenocarcinoma after successful radical surgical resection. ${ }^{29,30}$ Therefore, radical resection is the preferred treatment for this patient and similar cases. ${ }^{31,32}$

In conclusion, a SIT patient who developed a rare gallbladder adenosquamous carcinoma received a successful en-bloc surgical resection with no intraoperative vascular injuries. Proper imaging assessments, along with multidisciplinary cooperation, helped evaluate the visceral malformations, which eventually facilitated surgery success. The SIT population should receive regular surveillance to detect early and improve long-term survival in case malignancies occur.

\section{Abbreviations}

SIT, situs inversus totalis; CK7, cytokeratin 7; CK19, cytokeratin 19, CK5/6, cytokeratin 5/6; CT, computed tomography.

\section{Data Sharing Statement}

All data generated or analysed during this study are included in this article.

\section{Ethics Approval and Consent to Participate}

Ethics approval and consent was approved by Ethics Committee of First Affiliated Hospital, Zhejiang University School of Medicine.

\section{Consent for Publication}

Patient consent form had signed by patient.

\section{Acknowledgments}

We are very grateful to the patient for her support of clinical data collection.

\section{Author Contributions}

All authors made substantial contributions to conception and design, acquisition of data, or analysis and interpretation of data; took part in drafting the article or revising it critically for important intellectual content; agreed to submit to the current journal; gave final approval of the version to be published; and agree to be accountable for all aspects of the work.

\section{Funding}

This work was supported by financially by the National Natural Science Foundation of China (81972207, 81830089, 81530079, and 81502026), the Key Program of Medical Scientific Research Foundation of Zhejiang Province (2019C03019), the National High Technology Research and Development Program 863 of China (SS2015AA020405), Medical Health Science and Technology Project of Zhejiang Provincial Health Commission (2018KY406), and Zhejiang Provincial Natural Science Foundation (LQ16H180002 and LY18H160026).

\section{Disclosure}

There are no competing interests.

\section{References}

1. Lee SE, Kim HY, Jung SE, et al. Situs anomalies and gastrointestinal abnormalities. J Pediatr Surg. 2006;41(7):1237-1242. doi:10.1016/j. jpedsurg.2006.03.045

2. Kyuno D, Kimura Y, Imamura M, et al. Pancreaticoduodenectomy for biliary tract carcinoma with situs inversus totalis: difficulties and technical notes based on two cases. World J Surg Oncol. 2013;11 (1):312. doi:10.1186/1477-7819-11-312

3. Bartoloni L, Blouin JL, Pan Y, et al. Mutations in the DNAH11 (axonemal heavy chain dynein type 11) gene cause one form of situs inversus totalis and most likely primary ciliary dyskinesia. Proc Natl Acad Sci U S A. 2002;99(16):10282-10286. doi:10.1073/ pnas. 152337699

4. Reish O, Aspit L, Zouella A, et al. A homozygous nme7 mutation is associated with situs inversus totalis. Hum Mutat. 2016;37 (8):727-731. doi:10.1002/humu.22998

5. Afzelius BA. A human syndrome caused by immotile cilia. Science. 1976;193(4250):317-319. doi:10.1126/science.1084576

6. Kobus C, Targarona EM, Bendahan GE, et al. Laparoscopic surgery in situs inversus: a literature review and a report of laparoscopic sigmoidectomy for diverticulitis in situs inversus. Langenbecks Arch Surg. 2004;389(5):396-399. doi:10.1007/s00423-004-0500-0

7. Chaouch MA, Jerraya H, Dougaz MW, et al. A systematic review of laparoscopic cholecystectomy in situs inversus. J Investig Surg. 2021;34:324-333. doi:10.1080/08941939.2019.1622822

8. Mimae T, Nozaki I, Kurita A, et al. Esophagectomy via left thoracotomy for esophageal cancer with situs inversus totalis: report of a case. Surg Today. 2008;38(11):1044-1047. doi:10.1007/s00595-0083770-2

9. Maruyama Y, Horiuchi H, Okabe Y, et al. Perioperative challenges associated with a pancreaticoduodenectomy and distal pancreatectomy for pancreatic cancer in patients with situs inversus totalis: report of two cases. Surg Today. 2010;40(1):79-82. doi:10.1007/ s00595-009-4064-z

10. Ibrahim EM, Al-Idrissi H, Al-Farag A, et al. Situs inversus totalis with embryonal cell carcinoma of ovaries. Gynecol Oncol. 1984;18 (2):270-273. doi:10.1016/0090-8258(84)90036-2

11. Benson AB, D'Angelica MI, Abbott DE, et al. Guidelines insights: hepatobiliary cancers, version 2.2019. J Natl Compr Canc Netw. 2019;17(4):302-310. doi:10.6004/jnccn.2019.0019

12. Varga I, Galfiova P, Adamkov M, et al. Congenital anomalies of the spleen from an embryological point of view. Med Sci Monit. 2009;15: Ra269-Ra276.

13. Vetrini F, D'Alessandro LC, Akdemir ZC, et al. Bi-allelic mutations in PKD1L1 are associated with laterality defects in humans. Am J Hum Genet. 2016;99(4):886-893. doi:10.1016/j.ajhg.2016.07.011

14. Fonkalsrud EW, Tompkins R, Clatworthy HW Jr. Abdominal manifestations of situs inversus in infants and children. Arch Surg. 1966;92(5):791-795. doi:10.1001/archsurg.1966.01320230139025 
15. Moller JH, Nakib A, Anderson RC, et al. Congenital cardiac disease associated with polysplenia. A developmental complex of bilateral "left-sidedness". Circulation. 1967;36(5):789-799. doi:10.1161/01. CIR.36.5.789

16. Casey B, Devoto M, Jones KL, et al. Mapping a gene for familial situs abnormalities to human chromosome Xq24-q27.1. Nat Genet. 1993;5(4):403-407. doi:10.1038/ng1293-403

17. Wang J, Wang Q, Wei B, et al. Intronic polymorphisms in genes LRFN2 (rs2494938) and DNAH11 (rs2285947) are prognostic indicators of esophageal squamous cell carcinoma. BMC Med Genet. 2019;20(1):72. doi:10.1186/s12881-019-0796-9

18. Verma S, Bakshi D, Sharma V, et al. Genetic variants of DNAH11 and LRFN2 genes and their association with ovarian and breast cancer. Int J Gynaecol Obstet. 2020;148(1):118-122. doi:10.1002/ ijgo. 12997

19. Nöthe-Menchen T, Wallmeier J, Pennekamp P, et al. Randomization of left-right asymmetry and congenital heart defects: the role of DNAH5 in humans and mice. Circ Genom Precis Med. 2019;12 (11). doi:10.1161/CIRCGEN.119.002686.

20. Li F, Fang Z, Zhang J, et al. Identification of TRA2B-DNAH5 fusion as a novel oncogenic driver in human lung squamous cell carcinoma. Cell Res. 2016;26(10):1149-1164. doi:10.1038/cr.2016.111

21. Xiao WH, Qu XL, Li XM, et al. Identification of commonly dysregulated genes in colorectal cancer by integrating analysis of RNA-Seq data and qRT-PCR validation. Cancer Gene Ther. 2015;22(5):278-284. doi:10.1038/cgt.2015.20

22. Simons M, Gloy J, Ganner A, et al. Inversin, the gene product mutated in nephronophthisis type II, functions as a molecular switch between Wnt signaling pathways. Nat Genet. 2005;37(5):537-543. doi:10.1038/ng1552

23. Ireland $\mathrm{H}$, Kemp R, Houghton $\mathrm{C}$, et al. Inducible cre-mediated control of gene expression in the murine gastrointestinal tract: effect of loss of beta-catenin. Gastroenterology. 2004;126:1236-1246.
24. Maemura K, Natsugoe S, Takao S. Molecular mechanism of cholangiocarcinoma carcinogenesis. J Hepatobiliary Pancreat Sci. 2014;21 (10):754-760. doi:10.1002/jhbp.126

25. Misra S, Chaturvedi A, Misra NC, et al. Carcinoma of the gallbladder. Lancet Oncol. 2003;4(3):167-176. doi:10.1016/S14702045(03)01021-0

26. Albores-Saavedra J, Henson DE, Sobin LH. The WHO histological classification of tumors of the gallbladder and extrahepatic bile ducts. A commentary on the second edition. Cancer. 1992;70(2):410-414. doi:10.1002/1097-0142(19920715)70:2<410::AIDCNCR2820700207>3.0.CO;2-R

27. Henson DE, Albores-Saavedra J, Corle D. Carcinoma of the gallbladder. Histologic types, stage of disease, grade, and survival rates. Cancer. 1992;70(6):1493-1497. doi:10.1002/1097-0142(19920915) 70:6<1493::AID-CNCR2820700608>3.0.CO;2-U

28. Kim WS, Jang KT, Choi DW, et al. Clinicopathologic analysis of adenosquamous/squamous cell carcinoma of the gallbladder. J Surg Oncol. 2011;103(3):239-242. doi:10.1002/jso.21813

29. Roa JC, Tapia O, Cakir A, et al. Squamous cell and adenosquamous carcinomas of the gallbladder: clinicopathological analysis of 34 cases identified in 606 carcinomas. Mod Pathol. 2011;24 (8):1069-1078. doi:10.1038/modpathol.2011.68

30. Oohashi Y, Shirai Y, Wakai T, et al. Adenosquamous carcinoma of the gallbladder warrants resection only if curative resection is feasible. Cancer. 2002;94(11):3000-3005. doi:10.1002/cncr.10578

31. Guo CX, Chen W, Yao WY, et al. The first report of laparoscopic pancreaticoduodenectomy for primary duodenal carcinoma in a patient with situs inversus totalis: report of a rare case. Surg Laparosc Endosc Percutan Tech. 2019;29(3):e29-e33. doi:10.1097/ SLE.0000000000000659

32. Ito T, Saito M, Kobayashi Y, et al. [Two cases of digestive organ cancer in patients with situs inversus treated with laparoscopic surgery]. Gan to Kagaku Ryoho. 2015;42:2130-2132. [Japanese]
OncoTargets and Therapy

\section{Publish your work in this journal}

OncoTargets and Therapy is an international, peer-reviewed, open access journal focusing on the pathological basis of all cancers, potential targets for therapy and treatment protocols employed to improve the management of cancer patients. The journal also focuses on the impact of management programs and new therapeutic

\section{Dovepress}

agents and protocols on patient perspectives such as quality of life, adherence and satisfaction. The manuscript management system is completely online and includes a very quick and fair peer-review system, which is all easy to use. Visit http://www.dovepress.com/ testimonials.php to read real quotes from published authors. 